Ophthalmologica 1959;138:I-VI

\title{
Contents, Vol. 138, 1959
}

\section{Index}

Agarwal, L. P. and Malik, S. R. K.:

Post-operative Iris Prolapse. Its Cause and Management 133

Alberth, B.: $\quad$ Ein neues Verfahren zur Behandlung der Keratitis Herpetica ... 311

Arasimowicz, C. und Chwirot, R.:

Operativer Zugang zum Sehnerven beim Hunde 381

Benkö, E.: Objekt- und Farbengesichtsfeldeinengung bei chronischem Lärmschaden 449

Berardinis, E. de: The Presence of a Direct Oxidative Cycle in the Retina

Berardinis, E. de: vide Bonavolontã, A.

Best,F. : Beitrag zur Beziehung des Sensorischen zum Motorischen beim Sehen 183

Biró, I.: Recent Observations upon the Occurence of Hereditary Glaucoma 161

Bleeker, G. M. and Van Ommen, B.:

Early Treatment of Orbital Fractures

40

Bon,W. F.: On the Opacity of the Eye Lens Caused by Cooling 35

Bonavolontã, A., De Berardinis, E. and Bonavolontã, G.:

First Observations on Enzymatic Zonulolysis of Barraquer in Cata

ract Extraction 370

Bonavolontã, G.: vide Bonavolontã, A.

Bouman, M. A.: vide Brink, G. van den

Brink, G. van den and Bouman, M. A.:

Visual Acuity Depending on Spherical Correction 222

Bruckner, R.: Über Methoden longitudinaler Alternsforschung am Auge .... 59

Canossa, A.: vide Ricci, A.

Castro Correia, J.: vide Silva Pinto, M.

Cavka, V.: The Further Investigation of the Fronto-Orbital Centres for Intra ocular and Blood Pressure 413

Chwirot, R.: Über Papillenatrophien nach experimenteller Sehnervendurchschnei-

dung 436

Chwirot, R.: vide Arasimowicz, C.

Coimbra,A.: vide Silva Pinto, M.

Conrads, H.: Regenerative Veränderungen der Hornhautinnervation

Conrads, H. vide Wagner, K.

Darabos, G. and Vörösmarthy, D.:

Septum Canalis Nervi Optici. A Case Report 347

Dekking, H.M.: Demonstrations 230

Doesschate, G. ten:

Was Cezanne a forerunner of Luneburg? 456

Eggink, E. D.: Preliminary Report on Routine Tonometry in 1000 Patients over 
40 Years of Age 225

Fazakas,S.: Zusammenfassender Bericht über die sekundären Mykosen bei Erkrankungen des Augenlidrandes, der Bindehaut und der Hornhaut 108

Forgács, J.: vide Franceschetti, A.

Franceschetti, A. et Forgács, J.:

Aspect histologique de la dégénérescence limbique en ceinture

(«Weißer Limbusgürtel» de Vogt) et son analogie avec la dégénéres

cence primitive en bandelette de la cornée 393

Francois, J. et Neetens, A.:

L'engainement des veines rétiniennes dans la sclérose en plaques . 322

Gát, L. und Nagy, J.:

Durch Kalksalze verursachte Binde- und Hornhautläsionen bei Selbst-

verstümmelung $\quad 406$

Gessler,A.: Bestehen Beziehungen zwischen dem frühzeitigen Auftreten eines

Arcus lipoides corneae und Zirkulationsstörungen des Herzens, ins-

besondere dem Herzinfarkt? 118

Hamburg, A.: Some Investigations on the Cells of the Vitreous Body

81

Heinzen, H. und Würth,A.:

Die Photographie des Kammerwinkcls. (Ilierzu Tafel I) 214

Hradecký, F.: vide Svèrák, J.

Huysmans,J.: Anthrax of the Eyelids 236

Treatment of Diplopia due to Scar Tissue $\quad 237$

Correction of Excyclophoria 238

Illig, K. M.: Eine neuartige fistulierende Glaukomoperation

Jahnke, W.: Trachomerfahrungen im Orient .

422

Konstas, K. A.: Beobachtungen über den Akkommodationsexzess

Kozou§ek, V.: Beitrag zur Klinik der Flimmerperimetrie

Küchle, H. J.: Über Reaktionen und Regulationsmechanismen des intraocularen

Druckes 254

Lavergne, G.: Les modifications post-opératoires de la rigidité oculaire

Lasco, Fl.: vide Schmitzer, G.

Lichtenberg, R.: vide Schmitzer, G.

Malik, S. R. K.: vide Agarwll, L. P.

Mathur, S.P.: Amniotic Membrane in Corneal Ulcers

307

Nagy, J.: vide Gát, L.

Neetens, A.: vide Francois, J.

Nicolesco, M.: vide Schmitzer, G.

Norz,L.: vide Schmitzer, G.

Obniski, J.: vide Orlowsky, W. J.

Oey, K. L.: $\quad$ Muscle Transplantation as Additional Therapy of Concomitant Stra-

bism 431

Oksala, A.: The Echogram in Detachment of the Retina 350

Ommen, B. van: vide Bleeker, G. M.

Orlowski,W. J. and Obniski, J.:

Controlled Hypotension in Eye Surgery 264

Pantzer, L.: vide Schmitzer, G. 
Pau, H.: $\quad$ Die Operation der Netzhautablösung mit der Plombe

Peregrin, J.: vide Svërák, J.

Prijot, E. et Weekers, R.:

Contribution à $\Gamma$ étude de la rigidité de Гæil humain normal ... $\quad 1$

Ricci, A. et Canossa, A.:

L'ophtalmoscope électrique indirect de la clinique ophtalmologique

de Geneve 10

Scharf-Mayweg, S.:

Zum Thema der Schielbehandlung im Kindesalter 175

Schmidt, J. G. H.: Uber das Vorkommen von Neuraminsäure in verschiedenen Augen-

abschnitten des Rindes $\quad 314$

Schmitzer, G., Lichtenberg, R., Lasco,F., Pantzer, L., Norz, L. et Nicolesco, M.:

Les résultats de la ræntgenthérapie aux rayons durs, dans le traite-

ment des irido-cyclites 138

Schweitzer, N. M. J.:

A Glaucoma Department 233

Sebestyén, J.: Über die diffusen familiären Chorioretinalatrophien 336

Shapiro, D.: vide Stucchi, C.

Silva Pinto, M.; Castro Correia, J. et Coimbra,A.:

Alterations de la rétine provoquées par les petites insuffisances cir-

culatoires expérimentales chorio-rétiniennes 274

Stepanik, J.: Der Einfluß von Chlorothiazide (Diuril, Chlotride) auf den Augen-

druck des Menschen 361

Stucchi, C. et Shapiro, D.:

L'emploi de la Prazine en thérapeutique préparatoire pour la chi-

rurgie de la cataracte 146

Svèrák,J.; Peregrin, J. und Hradecký,F.:

ERG-Beitrag zum Metabolismus der Netzhaut. (Glukose-Test) . . 287

Valu, L.: $\quad$ Über das myopische Glaukom 302

Valu,L.: $\quad$ Canaliculodacryocystostomie 378

VelickÝ, G.: Contribution to the Study of Congenital Blindness 330

Vörösmabthy, D.: vide Darabos, G.

Wagner, K. und Conrads,H.:

Histologische Untersuchungen zur Genese der Periphlebitis retinae 399

Weekers, R.: vide Prijot, E.

Würth, A.: vide Heinzen, $H$.

Zagora, E.: Observations on the Evolution and Neurophysiology of Eye-Limbs

Coordination 241

GESELLSCHAFTSBERICHTE - SOCIETY TRANSACTIONS - SOCIÉTÉS

The Ophthalmological Society of the United Kingdom. Annual Con

gress - April 9th, 10th and 11th, 1959

151

Netherlands Ophthalmological Society. 141st Meeting in Groningen,

May 17th and 18th 1958 221

Zum 85. Geburtstag von Prof. Dr. J. Meller 320

Zum 60. Geburtstag von Prof. Dr. H. Goldmann

BUCHBESPRECHUNGEN - BOOK REVIEWS - LIVRES NOUVEAUX 
$75,154,239,316,388,459$

VARIA

$79,239,464$ 